\title{
ON AN INTEGRAL OPERATOR FROM THE ZYGMUND SPACE TO THE BLOCH-TYPE SPACE ON THE UNIT BALL
}

\author{
STEVO STEVIĆ \\ Mathematical Institute of the Serbian Academy of Science, Knez Mihailova 36/III, 11000 Beograd, Serbia \\ e-mail:sstevic@ptt.rs
}

(Received 5 April 2008; accepted 25 September 2008)

Abstract. In this paper, we introduce an integral operator on the unit ball $\mathbb{B} \subset \mathbb{C}^{n}$. The boundedness and compactness of the operator from the Zygmund space to the Bloch-type space $\mathcal{B}_{\mu}(\mathbb{B})$ or the little Bloch-type space $\mathcal{B}_{\mu, 0}(\mathbb{B})$ are investigated.

2000 Mathematics Subject Classification. Primary: 47B38; Secondary: 47B33.

1. Introduction. Let $\mathbb{B}=\mathbb{B}^{n}$ denote the unit ball in the complex vector space $\mathbb{C}^{n}$, $\partial \mathbb{B}=S$ its boundary, $\delta \mathbb{B}=\{z:|z| \leq \delta\}, \mathbb{D}=\mathbb{B}^{1}$ the unit disk in the complex plane $\mathbb{C}$, $H(\mathbb{B})$ the class of all analytic functions on $\mathbb{B}$. Let $z=\left(z_{1}, \ldots, z_{n}\right)$ and $w=\left(w_{1}, \ldots, w_{n}\right)$ be points in $\mathbb{C}^{n}$ and $\langle z, w\rangle=\sum_{k=1}^{n} z_{k} \bar{w}_{k}$. For $f \in H(\mathbb{B})$ with the Taylor expansion $f(z)=\sum_{|\beta| \geq 0} a_{\beta} z^{\beta}$, let

$$
\Re f(z)=\sum_{|\beta| \geq 0}|\beta| a_{\beta} z^{\beta}
$$

be the radial derivative of $f$, where $\beta=\left(\beta_{1}, \beta_{2}, \ldots, \beta_{n}\right)$ is a multi-index, $|\beta|=$ $\beta_{1}+\cdots+\beta_{n}$ and $z^{\beta}=z_{1}^{\beta_{1}} \ldots z_{n}^{\beta_{n}}$. It is well known (see, for example, [28]) that

$$
\Re f(z)=\sum_{j=1}^{n} z_{j} \frac{\partial f}{\partial z_{j}}(z)=\langle\nabla f(z), \bar{z}\rangle .
$$

A positive continuous function $\phi$ on $[0,1)$ is called normal [30] if there is $\delta \in[0,1)$ and $a$ and $b, 0<a<b$ such that

$$
\begin{aligned}
& \frac{\phi(r)}{(1-r)^{a}} \text { is decreasing on }[\delta, 1) \text { and } \lim _{r \rightarrow 1} \frac{\phi(r)}{(1-r)^{a}}=0 \\
& \frac{\phi(r)}{(1-r)^{b}} \text { is increasing on }[\delta, 1) \text { and } \lim _{r \rightarrow 1} \frac{\phi(r)}{(1-r)^{b}}=\infty .
\end{aligned}
$$

The Bloch-type space, denoted by $\mathcal{B}_{\mu}=\mathcal{B}_{\mu}(\mathbb{B})$, consists of all $f \in H(\mathbb{B})$ such that

$$
B_{\mu}(f)=\sup _{z \in \mathbb{B}} \mu(z)|\Re f(z)|<\infty,
$$

where $\mu(z)=\mu(|z|)$ and $\mu$ is normal on $[0,1)$. With the norm

$$
\|f\|_{\mathcal{B}_{\mu}}=|f(0)|+B_{\mu}(f)
$$

the Bloch-type space becomes a Banach space. 
The little Bloch-type space $\mathcal{B}_{\mu, 0}$ is a subspace of $\mathcal{B}_{\mu}$ consisting of those $f \in \mathcal{B}_{\mu}$ such that

$$
\lim _{|z| \rightarrow 1} \mu(z)|\Re f(z)|=0 .
$$

The $\alpha$-Bloch space $\mathcal{B}^{\alpha}$ is obtained for $\mu(z)=\left(1-|z|^{2}\right)^{\alpha}, \alpha \in(0, \infty)$ (see, e.g., $[\mathbf{1}, \mathbf{1 4}$, 27, 31, 35, 43, 45]). For $\alpha=1$ the space $\mathcal{B}^{1}=\mathcal{B}$ is the classical Bloch space.

Bloch-type spaces are usually defined by using the gradient of $f$ instead of its radial derivative. In $[40]$ it is shown that $\mathcal{B}_{\mu}$ consists of all $f \in H(\mathbb{B})$ such that

$$
\sup _{z \in \mathbb{B}} \mu(z)|\nabla f(z)|<\infty ;
$$

moreover, the following relationship holds

$$
\sup _{z \in \mathbb{B}} \mu(z)|\nabla f(z)| \asymp \sup _{z \in \mathbb{B}} \mu(z)|\Re f(z)| .
$$

For the case of $\mu(z)=\left(1-|z|^{2}\right)$, see, e.g., [4]. Also, it was proved that the little Bloch-type space is equivalent with the subspace of $\mathcal{B}_{\mu}$ consisting of all $f \in H(\mathbb{B})$ such that

$$
\lim _{|z| \rightarrow 1} \mu(z)|\nabla f(z)|=0 .
$$

Depending on a specific situation sometimes it is more appropriate to use one of the two definitions of the Bloch-type space. In this paper, we will use the first definition.

By $\mathcal{Z}$, we denote the class of all $f \in H(\mathbb{B}) \bigcap C(\overline{\mathbb{B}})$ such that

$$
\|f\|_{\mathcal{Z}}=\sup \frac{|f(\zeta+h)+f(\zeta-h)-2 f(\zeta)|}{|h|}<\infty,
$$

where the supremum is taken over all $\zeta \in \partial \mathbb{B}$ and $\zeta \pm h \in \partial \mathbb{B}$. From a known theorem (see [45, p. 261]), due to Zygmund for the case of the unit disk [6], and the Closed-Graph Theorem we know that $f \in \mathcal{Z}$ if and only if

$$
\sup _{z \in \mathbb{B}}\left(1-|z|^{2}\right)\left|\Re^{2} f(z)\right|<\infty
$$

moreover,

$$
\|f\|_{\mathcal{Z}} \asymp \sup _{z \in \mathbb{B}}\left(1-|z|^{2}\right)\left|\Re^{2} f(z)\right| .
$$

Therefore, we call $\mathcal{Z}$ the Zygmund class. The quantities in (3) are semi-norms, it is natural to add to them the quantity $|f(0)|+|\nabla f(0)|$ (correspondingly $|f(0)|)$, to obtain two equivalent norms on the Zygmund class. The second norm will be again denoted by $\|\cdot\|_{\mathcal{Z}}$. Zygmund class with this norm will be called the Zygmund space.

The little Zygmund space on $\mathbb{B}$, denoted by $\mathcal{Z}_{0}$, is the closed subspace of $\mathcal{Z}$ consisting of functions $f$ satisfying the following condition:

$$
\lim _{|z| \rightarrow 1}\left(1-|z|^{2}\right)\left|\Re^{2} f(z)\right|=0 .
$$


Let $\varphi$ be a non-constant analytic self-map of the unit ball. Associated with $\varphi$ is the composition operator $C_{\varphi}$ defined by $C_{\varphi} f=f \circ \varphi$ for $f \in H(\mathbb{B})$. If $u \in H(\mathbb{B})$ then by $u C_{\varphi} f(z)=u(z) f(\varphi(z))$ the weighted composition operator is defined. The main subject in the study of these operators is to describe operator theoretic properties of $C_{\varphi}$ and $u C_{\varphi}$ in terms of function theoretic properties of $\varphi$ (corresp. in terms of $\varphi$ and $u$ ). For some classical results see, for example, [5]. For some recent results mostly in the unit ball setting or Bloch-type spaces see, e.g., $[4,7,15-17,22-27,29,33,36,41,44]$ and the references therein.

In [19] (see also [38]) we introduced the following integral-type operator

$$
C_{\varphi}^{g}(f)(z)=\int_{0}^{z} f^{\prime}(\varphi(\zeta)) g(\zeta) d \zeta, \quad f \in H(\mathbb{D}), \quad z \in \mathbb{D}
$$

and investigated its boundedness and compactness on some spaces of holomorphic functions on the unit disk. One of the reasons for introducing operator (4) relies on the fact that similar operators naturally come from isometries of some function spaces (see, e.g., [8]).

It is a natural problem to introduce closely related operators to operator (4) in the unit ball settings and to study their function theoretic properties on spaces of holomorphic functions on the unit ball in terms of their inducing functions.

Assume that $g \in H(\mathbb{B})$ with $g(0)=0$ and $\varphi$ is a holomorphic self-map of $\mathbb{B}$. Motivated by (4) we define the following integral-type operator on the unit ball:

$$
I_{\varphi}^{g}(f)(z)=\int_{0}^{1} \Re f(\varphi(t z)) g(t z) \frac{d t}{t}, \quad f \in H(\mathbb{B}), \quad z \in \mathbb{B} .
$$

Recall that a linear operator $L: X \rightarrow Y$, where $X$ and $Y$ are Banach spaces, is compact if for every bounded sequence $\left(x_{k}\right)_{k \in \mathbb{N}}$ in $X$, the sequence $\left(L\left(x_{k}\right)\right)_{k \in \mathbb{N}}$ has a convergent subsequence.

Some characterizations of the boundedness and compactness of closely related integral-type operators in $\mathbb{C}^{n}$ can be found, for example, in $[\mathbf{3}, \mathbf{9 - 1 4}, \mathbf{1 8}, \mathbf{2 0}, \mathbf{2 1}, \mathbf{3 1}, 32$, 34, 37, 39, 40, 42] (see also the references therein).

Here, our aim is to study the boundedness and compactness of operator (5) from the Zygmund space to the Bloch-type space and the little Bloch-type space on the unit ball.

Throughout this paper, constants are denoted by $C$; they are positive and not necessarily the same at different occurrences. If we say that a function $\mu: \mathbb{C} \rightarrow[0, \infty)$ is normal we will assume that it is also radial, i.e., $\mu(z)=\mu(|z|), z \in \mathbb{B}$. The notation $a \preceq b$ means that there is a positive constant $C$ such that $a \leq C b$. We say that $a \asymp b$ if both $a \preceq b$ and $b \preceq a$ hold.

2. Auxiliary results. In this section, we quote several auxiliary results which will be used in the proofs of the main results in this paper.

The following lemma is fundamental in this paper. Closely related results can be found in the proof of Theorem 1 in [9] and [14, Lemma 1]. We give a proof of it for the completeness.

Lemma 1. Suppose that $f, g \in H(\mathbb{B}), g(0)=0$. Then

$$
\Re I_{\varphi}^{g}(f)(z)=\Re f(\varphi(z)) g(z) .
$$


Proof. Assume that the holomorphic function $\Re f(\varphi(z)) g(z)$ has the expansion $\sum_{\alpha} a_{\alpha} z^{\alpha}$. Note that the condition $g(0)=0$ implies $\alpha \neq 0$. Then

$$
\Re\left[I_{\varphi}^{g}(f)\right](z)=\Re \int_{0}^{1} \sum_{\alpha \neq 0} a_{\alpha}(t z)^{\alpha} \frac{d t}{t}=\Re\left(\sum_{\alpha \neq 0} \frac{a_{\alpha}}{|\alpha|} z^{\alpha}\right)=\sum_{\alpha} a_{\alpha} z^{\alpha},
$$

which is what we wanted to prove.

The following lemma follows by standard arguments (see, for example, the proofs of the corresponding results in $[\mathbf{5}, \mathbf{1 4}, \mathbf{3 2}-\mathbf{3 4}]$.

LEMMA 2. Suppose $\mu$ is normal, $g \in H(\mathbb{B}), g(0)=0$ and $\varphi$ is an analytic self-map of $\mathbb{B}$. Then $I_{\varphi}^{g}: \mathcal{Z}\left(\right.$ or $\left.\mathcal{Z}_{0}\right) \rightarrow \mathcal{B}_{\mu}$ is compact if and only if $I_{\varphi}^{g}: \mathcal{Z}\left(\right.$ or $\left.\mathcal{Z}_{0}\right) \rightarrow \mathcal{B}_{\mu}$ is bounded, and for any bounded sequence $\left(f_{k}\right)_{k \in \mathbb{N}}$ in $\mathcal{Z}\left(\right.$ or $\left.\mathcal{Z}_{0}\right)$ which converges to zero uniformly on compacts of $\mathbb{B}$ as $k \rightarrow \infty$, we have $\left\|I_{\varphi}^{g} f_{k}\right\|_{\mathcal{B}_{\mu}} \rightarrow 0$ as $k \rightarrow \infty$.

The proof of the next lemma is similar to the proof of Lemma 1 in [25], which concerns the case of $\mu(z)=\left(1-|z|^{2}\right)$ on the unit disk, hence it is also omitted (for the case of $\mu(z)=\left(1-|z|^{2}\right)^{\alpha}, z \in \mathbb{D}, \alpha \in(0, \infty)$ see [27]).

Lemma 3. Suppose $\mu$ is normal. A closed set $K$ in $\mathcal{B}_{\mu, 0}$ is compact if and only if it is bounded and satisfies

$$
\lim _{|z| \rightarrow 1} \sup _{f \in K} \mu(z)|\Re f(z)|=0 .
$$

REMARK 1. If in the formulation of Lemma 3 we omit the assumption $K$ is closed, then compact should be replaced by relatively compact.

The following lemma was proved in Theorem 7.11 of ref. [45].

Lemma 4. Suppose $f \in H(\mathbb{B})$. Then $f \in \mathcal{Z}$ if and only if $\partial f / \partial z_{k} \in \mathcal{B}$ for each $k \in$ $\{1, \ldots, n\}$.

Lemma 5. Suppose $f \in \mathcal{Z}$. Then

$$
Z_{1}(f):=\sup _{z \in \mathbb{B}}\left(1-|z|^{2}\right)\left|\Re^{2} f(z)\right| \asymp|\nabla f(0)|+\sup _{z \in \mathbb{B}}\left(1-|z|^{2}\right)\left|\nabla^{2} f(z)\right|=: Z_{2}(f) .
$$

Proof. First note that by Lemma 4 and the asymptotic relationship displayed in (1) with $\mu(z)=\left(1-|z|^{2}\right)$, we have that $Z_{1}(f)$ is finite if and only if $Z_{2}(f)$ is finite. Hence, the Zygmund class is equal with the class of all $f \in H(\mathbb{B})$ such that $Z_{2}(f)$ is finite.

It is easy to see that the quantities $Z_{1}(f)$ and $Z_{2}(f)$ are norms on the class $\mathcal{Z} / \mathbb{C}$. With each of these norms $\mathcal{Z} / \mathbb{C}$ becomes a Banach space. We show that these norms are equivalent on the space, that is, there is a positive constant $C$ such that

$$
Z_{1}(f) / C \leq Z_{2}(f) \leq C Z_{1}(f)
$$

To this end, by using the Closed-Graph Theorem to the identity operator (or its inverse)

$$
I:\left(\mathcal{Z} / \mathbb{C}, Z_{1}(f)\right) \rightarrow\left(\mathcal{Z} / \mathbb{C}, Z_{2}(f)\right),
$$

it is enough to prove that at least one of the inequalities in (7) holds. 
It is easy to see that

$$
\mathfrak{R}^{2} f(z)=\Re f(z)+\sum_{j=1}^{n} z_{j}^{2} \frac{\partial^{2} f}{\partial z_{j}^{2}}(z)+2 \sum_{j \neq k} z_{j} z_{k} \frac{\partial^{2} f}{\partial z_{j} \partial z_{k}}(z),
$$

which implies

$$
\left|\Re^{2} f(z)\right| \leq|z||\nabla f(z)|+\left|\nabla^{2} f(z)\right| .
$$

Further, for each $j \in\{1, \ldots, n\}$ we have

$$
\begin{aligned}
\left|\frac{\partial f}{\partial z_{j}}(z)-\frac{\partial f}{\partial z_{j}}(0)\right| & =\left|\int_{0}^{1}\left\langle\nabla \frac{\partial f}{\partial z_{j}}(t z), \bar{z}\right\rangle d t\right| \\
& \leq \int_{0}^{1}\left|\nabla^{2} f(t z)\right||z| d t \leq Z_{2}(f) \frac{1}{2} \ln \frac{1+|z|}{1-|z|}
\end{aligned}
$$

From (9) and some elementary inequalities it follows that

$$
|\nabla f(z)| \leq C\left(|\nabla f(0)|+Z_{2}(f) \ln \frac{1+|z|}{1-|z|}\right),
$$

for some positive constant $C$ independent of $f$.

From (8) and (10) we obtain

$$
\left|\Re^{2} f(z)\right| \leq C\left(|\nabla f(0)|+\left|\nabla^{2} f(z)\right|+Z_{2}(f) \ln \frac{1+|z|}{1-|z|}\right) .
$$

Multiplying (11) by $\left(1-|z|^{2}\right)$ and taking the supremum in such obtained inequality over $z \in \mathbb{B}$ and using the fact that

$$
\sup _{x \in[0,1)}\left(1-x^{2}\right) \ln \frac{1+x}{1-x}<\infty
$$

we easily obtain $Z_{1}(f) \leq C Z_{2}(f)$, as desired.

Lemma 6. Suppose that $f \in \mathcal{Z}$. Then there is a positive constant $C$ independent off such that

$$
|\nabla f(z)| \leq C\|f\|_{\mathcal{Z}} \ln \frac{e}{1-|z|^{2}}
$$

Proof. In [31, Lemma 2.2], among others, we proved that there is a positive constant $C$ independent of $f$ such that for every $f \in \mathcal{B}$,

$$
|f(z)| \leq C\|f\|_{\mathcal{B}} \ln \frac{e}{1-|z|^{2}} .
$$

Applying (13) to the functions $\frac{\partial f}{\partial z_{l}}, l \in\{1, \ldots, n\}$, which belong to $\mathcal{B}$ in view of Lemma 4, using the asymptotics (1) with $\mu(z)=\left(1-|z|^{2}\right)$ and some elementary inequalities we 
obtain

$$
|\nabla f(z)| \leq C\left(|\nabla f(0)|+\sup _{w \in \mathbb{B}}\left(1-|w|^{2}\right)\left|\nabla^{2} f(w)\right|\right) \ln \frac{e}{1-|z|^{2}} .
$$

Since by Lemma 5

$$
|\nabla f(0)|+\sup _{w \in \mathbb{B}}\left(1-|w|^{2}\right)\left|\nabla^{2} f(w)\right| \preceq\|f\|_{\mathcal{Z}}
$$

we obtain (12).

3. The boundedness and compactness of $I_{\varphi}^{g}: \mathcal{Z}\left(\right.$ or $\left.\mathcal{Z}_{0}\right) \rightarrow \mathcal{B}_{\mu}\left(\right.$ or $\left.\mathcal{B}_{\mu, 0}\right)$. In this section, we prove the main results of this paper, namely, we characterize the boundedness and compactness of the operator $I_{\varphi}^{g}: \mathcal{Z}\left(\right.$ or $\left.\mathcal{Z}_{0}\right) \rightarrow \mathcal{B}_{\mu}\left(\right.$ or $\left.\mathcal{B}_{\mu, 0}\right)$.

THEOREM 1. Suppose $\mu$ is normal, $g \in H(\mathbb{B}), g(0)=0$ and $\varphi$ is an analytic self-map of $\mathbb{B}$. Then $I_{\varphi}^{g}: \mathcal{Z}\left(\right.$ or $\left.\mathcal{Z}_{0}\right) \rightarrow \mathcal{B}_{\mu}$ is bounded if and only if

$$
\sup _{z \in \mathbb{B}} \mu(z)|g(z)||\varphi(z)|<\infty
$$

and

$$
K:=\sup _{z \in \mathbb{B}} \mu(z)|g(z)||\varphi(z)| \ln \frac{1}{1-|\varphi(z)|^{2}}<\infty .
$$

Proof. First assume that $I_{\varphi}^{g}: \mathcal{Z}\left(\right.$ or $\left.\mathcal{Z}_{0}\right) \rightarrow \mathcal{B}_{\mu}$ is bounded.

By using the test functions given by

$$
f_{l}(z)=z_{l} \in \mathcal{Z}_{0}, \quad l \in\{1, \ldots, n\},
$$

we obtain $I_{\varphi}^{g} f_{l} \in \mathcal{B}_{\mu}$ for $l \in\{1, \ldots, n\}$, that is,

$$
\left\|I_{\varphi}^{g} f_{l}\right\|_{\mathcal{B}_{\mu}}=\sup _{z \in \mathbb{B}} \mu(z)|g(z)|\left|\varphi_{l}(z)\right|<\infty,
$$

for each $l \in\{1, \ldots, n\}$, and consequently

$$
\sup _{z \in \mathbb{B}} \mu(z)|g(z)||\varphi(z)| \leq \sum_{l=1}^{n} \sup _{z \in \mathbb{B}} \mu(z)\left|g(z) \| \varphi_{l}(z)\right|<\infty .
$$

Hence, condition (14) holds.

Set

$$
h(z)=(z-1)\left[\left(1+\ln \frac{1}{1-z}\right)^{2}+1\right], \quad z \in \mathbb{D}
$$

and

$$
h_{a}(z)=\frac{h(\langle z, a\rangle)}{|a|}\left(\ln \frac{1}{1-|a|^{2}}\right)^{-1}, \quad z \in \mathbb{B},
$$

for $a \in \mathbb{B}$ such that $|a|>\sqrt{1-1 / e}$. 
We have

$$
\Re h_{a}(z)=\frac{\langle z, a\rangle}{|a|}\left(\ln \frac{1}{1-\langle z, a\rangle}\right)^{2}\left(\ln \frac{1}{1-|a|^{2}}\right)^{-1}
$$

and

$$
\mathfrak{R}^{2} h_{a}(z)=\Re h_{a}(z)+\frac{2\langle z, a\rangle^{2}}{|a|(1-\langle z, a\rangle)}\left(\ln \frac{1}{1-\langle z, a\rangle}\right)\left(\ln \frac{1}{1-|a|^{2}}\right)^{-1} .
$$

Thus, for $\sqrt{1-1 / e}<|a|<1$, we obtain

$$
M_{1}=\sup _{\sqrt{1-1 / e}<|a|<1}\left\|h_{a}\right\|_{\mathcal{Z}}<\infty
$$

Moreover, we have that $h_{a} \in \mathcal{Z}_{0}$, for $\sqrt{1-1 / e}<|a|<1$.

Further, from the boundedness of $I_{\varphi}^{g}: \mathcal{Z}\left(\right.$ or $\left.\mathcal{Z}_{0}\right) \rightarrow \mathcal{B}_{\mu}$ we have

$$
\begin{aligned}
M_{1}\left\|I_{\varphi}^{g}\right\|_{\mathcal{Z} \rightarrow \mathcal{B}_{\mu}} & \geq\left\|h_{\varphi(a)}\right\|_{\mathcal{Z}}\left\|I_{\varphi}^{g}\right\|_{\mathcal{Z} \rightarrow \mathcal{B}_{\mu}} \geq\left\|I_{\varphi}^{g} h_{\varphi(a)}\right\|_{\mathcal{B}_{\mu}} \\
& =\sup _{z \in \mathbb{B}} \mu(z)\left|g(z) \Re h_{\varphi(a)}(\varphi(z))\right| \\
& \geq \mu(a)\left|g(a) \|\left\langle\nabla h_{\varphi(a)}(\varphi(a)), \overline{\varphi(a)}\right\rangle\right| \\
& =\mu(a)|g(a) \| \varphi(a)| \ln \frac{1}{1-|\varphi(a)|^{2}} .
\end{aligned}
$$

From (18) it follows that

$$
\sup _{\sqrt{1-1 / e}<|\varphi(z)|<1} \mu(z)|g(z)||\varphi(z)| \ln \frac{1}{1-|\varphi(z)|^{2}}<\infty .
$$

On the other hand (14) yields

$$
\sup _{|\varphi(z)| \leq \sqrt{1-1 / e}} \mu(z)|g(z)||\varphi(z)| \ln \frac{1}{1-|\varphi(z)|^{2}} \leq \sup _{z \in \mathbb{B}} \mu(z)|g(z)||\varphi(z)|<\infty .
$$

From (19) and (20), we obtain (15) as claimed.

Now assume that (14) and (15) hold. By Lemmas 1 and 6 and (12) it follows that

$$
\begin{aligned}
\mu(z)\left|\Re\left(I_{\varphi}^{g} f\right)(z)\right| & =\mu(z)|\Re f(\varphi(z))||g(z)| \\
& \leq \mu(z)|\nabla f(\varphi(z))||\varphi(z)||g(z)| \\
& \leq C\|f\|_{\mathcal{Z}} \mu(z)|g(z) \| \varphi(z)| \ln \frac{e}{1-|\varphi(z)|^{2}}
\end{aligned}
$$

for every $z \in \mathbb{B}$ and $f \in \mathcal{Z}$.

From this, (14), (15) and since $I_{\varphi}^{g} f(0)=0$, it follows that $I_{\varphi}^{g}: \mathcal{Z}\left(\right.$ or $\left.\mathcal{Z}_{0}\right) \rightarrow \mathcal{B}_{\mu}$ is bounded, completing the proof of the theorem.

THEOREM 2. Suppose $\mu$ is normal, $g \in H(\mathbb{B}), g(0)=0$ and $\varphi$ is an analytic self-map of $\mathbb{B}$. Then $I_{\varphi}^{g}: \mathcal{Z}\left(\right.$ or $\left.\mathcal{Z}_{0}\right) \rightarrow \mathcal{B}_{\mu}$ is compact if and only if $I_{\varphi}^{g}: \mathcal{Z}\left(\right.$ or $\left.\mathcal{Z}_{0}\right) \rightarrow \mathcal{B}_{\mu}$ is bounded 
and

$$
\lim _{|\varphi(z)| \rightarrow 1} \mu(z)|g(z)||\varphi(z)| \ln \frac{e}{1-|\varphi(z)|^{2}}=0 .
$$

Proof. First suppose that $I_{\varphi}^{g}: \mathcal{Z}\left(\right.$ or $\left.\mathcal{Z}_{0}\right) \rightarrow \mathcal{B}_{\mu}$ is compact. Clearly $I_{\varphi}^{g}: \mathcal{Z}\left(\right.$ or $\left.\mathcal{Z}_{0}\right) \rightarrow$ $\mathcal{B}_{\mu}$ is bounded.

Now we prove that (21) holds. Let $\left(z_{k}\right)_{k \in \mathbb{N}}$ be a sequence in $\mathbb{B}$ such that $\left|\varphi\left(z_{k}\right)\right| \rightarrow 1$ as $k \rightarrow \infty$ (if such a sequence does not exist (21) is vacuously satisfied). Set

$$
\hat{h}_{k}(z)=h_{\varphi\left(z_{k}\right)}(z), \quad k \in \mathbb{N},
$$

where $h_{a}$ is defined in (17). From the proof of Theorem 1 we see that $\sup _{k \in \mathbb{N}}\left\|\hat{h}_{k}\right\|_{\mathcal{Z}} \leq C$. Moreover, $\hat{h}_{k}$ converges to zero uniformly on compacts of $\mathbb{B}$ as $k \rightarrow \infty$. Also note that

$$
\mathfrak{R} h_{a}(a)=|a| \ln \frac{1}{1-|a|^{2}},
$$

for each $a \in \mathbb{B} \backslash\{0\}$.

By using Lemma 2, it follows that $\left\|I_{\varphi}^{g} \hat{h}_{k}\right\|_{\mathcal{B}_{\mu}} \rightarrow 0$ as $k \rightarrow \infty$. Since

$$
\begin{aligned}
\left\|I_{\varphi}^{g} \hat{h}_{k}\right\|_{\mathcal{B}_{\mu}} & =\sup _{z \in \mathbb{B}} \mu(z)\left|\Re\left(I_{\varphi}^{g} \hat{h}_{k}\right)(z)\right| \\
& \geq \mu\left(z_{k}\right)\left|g\left(z_{k}\right)\right|\left|\Re \hat{h}_{k}\left(\varphi\left(z_{k}\right)\right)\right| \\
& =\mu\left(z_{k}\right)\left|g\left(z_{k}\right)\right|\left|\varphi\left(z_{k}\right)\right| \ln \frac{1}{1-\left|\varphi\left(z_{k}\right)\right|^{2}},
\end{aligned}
$$

we obtain

$$
\lim _{k \rightarrow \infty} \mu\left(z_{k}\right)\left|g\left(z_{k}\right)\right|\left|\varphi\left(z_{k}\right)\right| \ln \frac{1}{1-\left|\varphi\left(z_{k}\right)\right|^{2}}=0,
$$

which along with the assumption $\left|\varphi\left(z_{k}\right)\right| \rightarrow 1$ as $k \rightarrow \infty$, obviously implies

$$
\lim _{k \rightarrow \infty} \mu\left(z_{k}\right)\left|g\left(z_{k}\right)\right|\left|\varphi\left(z_{k}\right)\right|=0 .
$$

From the last two limits, (21) follows.

Now assume that $I_{\varphi}^{g}: \mathcal{Z}$ (or $\left.\mathcal{Z}_{0}\right) \rightarrow \mathcal{B}_{\mu}$ is bounded and condition (21) holds. From the boundedness of $I_{\varphi}^{g}$ we know that condition (14) holds. Let $\left(f_{k}\right)_{k \in \mathbb{N}}$ be a sequence in $\mathcal{Z}\left(\right.$ or $\mathcal{Z}_{0}$ ) such that $\sup _{k \in \mathbb{N}}\left\|f_{k}\right\|_{\mathcal{Z}}=: M<\infty$ and $f_{k} \rightarrow 0$ uniformly on compacts of $\mathbb{B}$ as $k \rightarrow \infty$. By (21), we have that for every $\varepsilon>0$, there is a $\delta \in(0,1)$, such that

$$
\mu(z)|g(z) \| \varphi(z)| \ln \frac{e}{1-|\varphi(z)|^{2}}<\frac{\varepsilon}{M},
$$

whenever $\delta<|\varphi(z)|<1$.

By using Lemmas 1 and 6 and estimates (12) and (23), we obtain

$$
\begin{aligned}
\left\|I_{\varphi}^{g} f_{k}\right\|_{\mathcal{B}_{\mu}} & =\sup _{z \in \mathbb{B}} \mu(z)\left|g(z) \Re f_{k}(\varphi(z))\right| \\
& \leq \sup _{\{z \in \mathbb{B}:|\varphi(z)| \leq \delta\}} \mu(z)|g(z)|\left|\Re f_{k}(\varphi(z))\right|+\sup _{\{z \in \mathbb{B}: \delta<|\varphi(z)|<1\}} \mu(z)|g(z)|\left|\Re f_{k}(\varphi(z))\right|
\end{aligned}
$$


INTEGRAL OPERATOR FROM ZYGMUND SPACE TO BLOCH-TYPE SPACE 283

$$
\begin{aligned}
& \leq\|g \varphi\|_{H_{\mu}^{\infty}} \sup _{w \in \delta \mathbb{B}}\left|\nabla f_{k}(w)\right|+C\left\|f_{k}\right\|_{\mathcal{Z}} \sup _{\{z \in \mathbb{B}: \delta<|\varphi(z)|<1\}} \mu(z)|g(z) \| \varphi(z)| \ln \frac{e}{1-|\varphi(z)|^{2}} \\
& \leq\|g \varphi\|_{H_{\mu}^{\infty}} \sup _{w \in \delta \mathbb{B}}\left|\nabla f_{k}(w)\right|+C \varepsilon,
\end{aligned}
$$

where

$$
\|g \varphi\|_{H_{\mu}^{\infty}}:=\sup _{z \in \mathbb{B}} \mu(z)|g(z) \| \varphi(z)|,
$$

which is finite in light of (14).

Since $\left(f_{k}\right)_{k \in \mathbb{N}}$ is a sequence converging to zero on compacts of $\mathbb{B}$ as $k \rightarrow \infty$, then by Cauchy's estimate the sequence $\left(\left|\nabla f_{k}\right|\right)_{k \in \mathbb{N}}$ also converges to zero on compacts of $\mathbb{B}$ as $k \rightarrow \infty$. In particular, it follows that

$$
\lim _{k \rightarrow \infty} \sup _{w \in \delta \mathbb{B}}\left|\nabla f_{k}(w)\right|=0 .
$$

Letting $k \rightarrow \infty$ in (24) and using (25) we obtain

$$
\limsup _{k \rightarrow \infty}\left\|I_{\varphi}^{g} f_{k}\right\|_{\mathcal{B}_{\mu}} \leq C \varepsilon
$$

Since $\varepsilon$ is an arbitrary positive number, it follows that the last limit is equal to zero. Employing Lemma 2, the implication follows.

THEOREM 3. Suppose $\mu$ is normal, $g \in H(\mathbb{B}), g(0)=0$ and $\varphi$ is an analytic self-map of $\mathbb{B}$. Then $I_{\varphi}^{g}: \mathcal{Z}\left(\right.$ or $\left.\mathcal{Z}_{0}\right) \rightarrow \mathcal{B}_{\mu, 0}$ is bounded if and only if

$$
\lim _{|z| \rightarrow 1} \mu(z)|g(z)||\varphi(z)|=0
$$

and

$$
\lim _{|\varphi(z)| \rightarrow 1} \mu(z)|g(z)||\varphi(z)| \ln \frac{1}{1-|\varphi(z)|^{2}}=0
$$

Proof. Assume that (26) and (27) hold. By (27) we have that for every $\varepsilon>0$ there exists an $r \in(0,1)$ such that

$$
\mu(z)|g(z)||\varphi(z)| \ln \frac{1}{1-|\varphi(z)|^{2}}<\varepsilon,
$$

when $r<|\varphi(z)|<1$.

On the other hand, from $(26)$, there exists a $\rho \in(0,1)$ such that

$$
\mu(z)|g(z)||\varphi(z)|<\varepsilon / \ln \frac{1}{1-r^{2}},
$$

when $\rho<|z|<1$.

Therefore, when $\rho<|z|<1$ and $r<|\varphi(z)|<1$, from (28) we have

$$
\mu(z)|g(z)||\varphi(z)| \ln \frac{1}{1-|\varphi(z)|^{2}}<\varepsilon
$$


If $\rho<|z|<1$ and $|\varphi(z)| \leq r$, from (29) we obtain

$$
\mu(z)|g(z)||\varphi(z)| \ln \frac{1}{1-|\varphi(z)|^{2}}<\mu(z)|g(z)||\varphi(z)| \ln \frac{1}{1-r^{2}}<\varepsilon .
$$

Combining (30) and (31), we obtain

$$
\lim _{|z| \rightarrow 1} \mu(z)|g(z)||\varphi(z)| \ln \frac{1}{1-|\varphi(z)|^{2}}=0 .
$$

From (26) and (32), by using the Maximum Modulus Theorem and Theorem 1 the boundedness of $I_{\varphi}^{g}: \mathcal{Z}\left(\right.$ or $\left.\mathcal{Z}_{0}\right) \rightarrow \mathcal{B}_{\mu}$ follows.

By Lemma 1 and the estimate in (12), we have

$$
\mu(z)\left|\Re\left(I_{\varphi}^{g} f\right)(z)\right| \leq C\|f\|_{\mathcal{Z}} \mu(z)|g(z) \| \varphi(z)|\left(1+\ln \frac{1}{1-|\varphi(z)|^{2}}\right),
$$

for every $f \in \mathcal{Z}$ (or $\left.\mathcal{Z}_{0}\right)$.

By using (26) and (32) in inequality (33), it follows that for each $f \in \mathcal{Z}$ (or $\mathcal{Z}_{0}$ ), $I_{\varphi}^{g} f \in \mathcal{B}_{\mu, 0}$, that is, $I_{\varphi}^{g}(\mathcal{Z}) \subseteq \mathcal{B}_{\mu, 0}\left(\right.$ or $\left.I_{\varphi}^{g}\left(\mathcal{Z}_{0}\right) \subseteq \mathcal{B}_{\mu, 0}\right)$. Since $\mathcal{B}_{\mu, 0}$ is a closed subset of $\mathcal{B}_{\mu}$ the boundedness of $I_{\varphi}^{g}: \mathcal{Z}$ (or $\left.\mathcal{Z}_{0}\right) \rightarrow \mathcal{B}_{\mu, 0}$ follows.

Conversely, suppose that $I_{\varphi}^{g}: \mathcal{Z}\left(\right.$ or $\left.\mathcal{Z}_{0}\right) \rightarrow \mathcal{B}_{\mu, 0}$ is bounded.

Employing the test functions in (16) we obtain $I_{\varphi}^{g} f_{l} \in \mathcal{B}_{\mu, 0}, l \in\{1, \ldots, n\}$, that is,

$$
\mu(z)\left|I_{\varphi}^{g} f_{l}(z)\right|=\mu(z)|g(z)|\left|\varphi_{l}(z)\right| \rightarrow 0, \quad \text { as }|z| \rightarrow 1
$$

for each $l \in\{1, \ldots, n\}$ and consequently

$$
\lim _{|z| \rightarrow 1} \mu(z)|g(z)||\varphi(z)|=0 .
$$

Hence, condition (26) holds.

If $\|\varphi\|_{\infty}<1$, then condition (27) is vacuously satisfied. Hence, assume $\|\varphi\|_{\infty}=1$, and assume that condition (27) does not hold. If it would, then $\varepsilon_{0}>0$ and a sequence $\left(z_{k}\right)_{k \in \mathbb{N}} \in \mathbb{B}$ would exist, such that $\lim _{k \rightarrow \infty}\left|\varphi\left(z_{k}\right)\right|=1$ and

$$
\mu\left(z_{k}\right)\left|g\left(z_{k}\right)\right|\left|\varphi\left(z_{k}\right)\right| \ln \frac{1}{1-\left|\varphi\left(z_{k}\right)\right|^{2}} \geq \varepsilon_{0}>0
$$

for sufficiently large $k$.

We may also assume that $\varphi\left(z_{k}\right) \rightarrow(1,0, \ldots, 0)$ as $k \rightarrow \infty$ and

$$
\frac{1-\left|\varphi_{1}\left(z_{k-1}\right)\right|}{2}>1-\left|\varphi_{1}\left(z_{k}\right)\right|, \quad k \in \mathbb{N} \text {. }
$$

Then, for every non-negative integer $s$ there is at most one $z_{k}$ such that $1-\frac{1}{2^{s}} \leq$ $\left|\varphi_{1}\left(z_{k}\right)\right|<1-\frac{1}{2^{(s+1)}}$. Hence, there is an $m_{0} \in \mathbb{N}$ such that for any Carleson window

$$
Q=\left\{r e^{i \theta}|0<1-r<l(Q),| \theta-\theta_{0} \mid<l(Q)\right\}
$$

and $s \in \mathbb{N}$, there are at most $m_{0}$ elements in

$$
\left\{\varphi_{1}\left(z_{k}\right) \in Q\left|2^{-(s+1)} l(Q)<1-\right| \varphi_{1}\left(z_{k}\right) \mid<2^{-s} l(Q)\right\} .
$$


Therefore, $\left(\varphi_{1}\left(z_{k}\right)\right)_{k \in \mathbb{N}}$ is an interpolating sequence for $\mathcal{B}(\mathbb{D})$ in sense of [2].

By [2], there is a $p \in \mathcal{B}(\mathbb{D})$ such that

$$
p\left(\varphi_{1}\left(z_{k}\right)\right)=\ln \frac{1}{1-\left|\varphi_{1}\left(z_{k}\right)\right|^{2}}, \quad k \in \mathbb{N} .
$$

Let

$$
F(z)=F\left(z_{1}\right)=\int_{0}^{1} \frac{p\left(t z_{1}\right)-p(0)}{t} d t .
$$

Then from definitions of Bloch and Zygmund functions, we see that $F \in \mathcal{Z}$.

By Lemma 1, some simple calculation and (26), we obtain

$$
\begin{aligned}
\mu\left(z_{k}\right)\left|\Re\left(I_{\varphi}^{g} F\right)\left(z_{k}\right)\right| & =\mu\left(z_{k}\right)\left|g\left(z_{k}\right)\right|\left|\Re F\left(\varphi\left(z_{k}\right)\right)\right| \\
& =\mu\left(z_{k}\right)\left|g\left(z_{k}\right)\right|\left|\varphi_{1}\left(z_{k}\right)\right|\left|p\left(\varphi_{1}\left(z_{k}\right)\right)-p(0)\right| \\
& \asymp \mu\left(z_{k}\right)\left|g\left(z_{k}\right)\right|\left|\varphi_{1}\left(z_{k}\right)\right| \ln \frac{1}{1-\left|\varphi_{1}\left(z_{k}\right)\right|^{2}} \\
& \geq C \mu\left(z_{k}\right)\left|g\left(z_{k}\right)\right|\left|\varphi\left(z_{k}\right)\right| \ln \frac{1}{1-\left|\varphi\left(z_{k}\right)\right|^{2}} \\
& \geq C \varepsilon_{0}>0
\end{aligned}
$$

for sufficiently large $k$.

Since $\lim _{k \rightarrow \infty}\left|\varphi\left(z_{k}\right)\right|=1$ implies that $\lim _{k \rightarrow \infty}\left|z_{k}\right|=1$, from the above inequality we obtain that $I_{\varphi}^{g} f \notin \mathcal{B}_{\mu, 0}$, which is a contradiction.

THEOREM 4. Suppose $\mu$ is normal, $g \in H(\mathbb{B}), g(0)=0$ and $\varphi$ is an analytic self-map of $\mathbb{B}$. Then $I_{\varphi}^{g}: \mathcal{Z}\left(\right.$ or $\left.\mathcal{Z}_{0}\right) \rightarrow \mathcal{B}_{\mu, 0}$ is compact if and only if

$$
\lim _{|z| \rightarrow 1} \mu(z)|g(z)||\varphi(z)| \ln \frac{e}{1-|\varphi(z)|^{2}}=0 .
$$

Proof. Assume that (34) holds. Then (14) and (15) hold. By Lemma 1 and (12) we have

$$
\mu(z)\left|\Re\left(I_{\varphi}^{g} f\right)(z)\right| \leq C\|f\|_{\mathcal{Z}} \mu(z)|g(z)||\varphi(z)| \ln \frac{e}{1-|\varphi(z)|^{2}} .
$$

From this, (14) and (15) it follows that $I_{\varphi}^{g}\left(\left\{f:\|f\|_{\mathcal{Z}} \leq 1\right\}\right)$ (or $\left.I_{\varphi}^{g}\left(\left\{f \in \mathcal{Z}_{0}:\|f\|_{\mathcal{Z}} \leq 1\right\}\right)\right)$ is a bounded set in $\mathcal{B}_{\mu}$. Moreover, since (34) holds it is bounded in $\mathcal{B}_{\mu, 0}$. Taking the supremum in (35) over the unit ball of the space $\mathcal{Z}$ (or $\mathcal{Z}_{0}$ ) then letting $|z| \rightarrow 1$, we obtain

$$
\lim _{|z| \rightarrow 1} \sup _{\left.\|f\|_{\mathcal{Z}(o r} Z_{0}\right) \leq 1} \mu(z)\left|\Re\left(I_{\varphi}^{g} f\right)(z)\right|=0 .
$$

From (36) and by using Lemma 3 the compactness of the operator $I_{\varphi}^{g}: \mathcal{Z}\left(\right.$ or $\left.\mathcal{Z}_{0}\right) \rightarrow$ $\mathcal{B}_{\mu, 0}$ follows.

Now assume that $I_{\varphi}^{g}: \mathcal{Z}\left(\right.$ or $\left.\mathcal{Z}_{0}\right) \rightarrow \mathcal{B}_{\mu, 0}$ is compact. Then $I_{\varphi}^{g}: \mathcal{Z}\left(\right.$ or $\left.\mathcal{Z}_{0}\right) \rightarrow \mathcal{B}_{\mu, 0}$ is bounded, hence by Theorem 3 conditions (26) and (27) hold. By the proof of Theorem 3 we have that (32) holds and consequently (34), as desired. 


\section{REFERENCES}

1. K. Avetisyan, Hardy Bloch-type spaces and lacunary series on the polydisk, Glasgow $J$. Math. 49 (2007), 345-356.

2. B. Boe and A. Nikolau, Interpolation by functions in the Bloch space, J. Anal. Math. 94 (2004), 171-194.

3. D. C. Chang, S. Li and S. Stević, On some integral operators on the unit polydisk and the unit ball, Taiwanese J. Math. 11(5) (2007), 1251-1286.

4. D. Clahane and S. Stević, Norm equivalence and composition operators between Bloch/Lipschitz spaces of the unit ball J. Inequal. Appl. 2006 (2006), 11 pp. (Article ID 61018).

5. C. Cowen and B. MacCluer, Composition operators on spaces of analytic functions (Studies in Advanced Mathematics, CRC Press, Boca Raton, 1995).

6. P. Duren, Theory of $H^{p}$ spaces (Academic press, New York, 1973).

7. X. Fu and X. Zhu, Weighted composition operators on some weighted spaces in the unit ball, Abstr. Appl. Anal. 2008 (2008), 8 pp. (Article ID 605807).

8. W. Hornor and J. E. Jamison, Isometries of some Banach spaces of analytic functions, Integral Equations Operator Theory 41 (2001), 401-425.

9. Z. Hu, Extended Cesàro operators on mixed-norm spaces, Proc. Amer. Math. Soc. 131(7) (2003), 2171-2179.

10. Z. Hu, Extended Cesàro operators on the Bloch space in the unit ball of $\mathbb{C}^{n}$, Acta Math. Sci. Ser. B Engl. Ed. 23(4) (2003), 561-566.

11. Z. Hu, Extended Cesàro operators on Bergman spaces, J. Math. Anal. Appl. 296 (2004), 435-454.

12. S. Li and S. Stević, Integral type operators from mixed-norm spaces to $\alpha$-Bloch spaces, Integral Transform. Spec. Funct. 18(7) (2007), 485-493.

13. S. Li and S. Stević, Riemann-Stieltjes operators on Hardy spaces in the unit ball of $\mathbb{C}^{n}$, Bull. Belg. Math. Soc. Simon Stevin 14 (2007), 621-628.

14. S. Li and S. Stević, Riemann-Stieltjes type integral operators on the unit ball in $\mathbb{C}^{n}$, Complex Variables Elliptic Equations 52(6) (2007), 495-517.

15. S. Li and S. Stević, Weighted composition operators from Bergman-type spaces into Bloch spaces, Proc. Indian Acad. Sci. Math. Sci. 117(3) (2007), 371-385.

16. S. Li and S. Stević, Weighted composition operators from $\alpha$-Bloch space to $H^{\infty}$ on the polydisk, Numer. Funct. Anal. Optimization 28 (7) (2007), 911-925.

17. S. Li and S. Stević, Weighted composition operators from $H^{\infty}$ to the Bloch space on the polydisc, Abstr. Appl. Anal. 2007 (2007), 12 pp. (Article ID 48478).

18. S. Li and S. Stević, Compactness of Reimann-Stieltjes operators between $F(p, q, s)$ and $\alpha$-Bloch spaces, Publ. Math. Debrecen 72(1-2) (2008), 111-128.

19. S. Li and S. Stević, Generalized composition operators on Zygmund spaces and Bloch type spaces, J. Math. Anal. Appl. 338 (2008), 1282-1295.

20. S. Li and S. Stević, Riemann-Stieltjes operators between mixed norm spaces, Indian J. Math. 50(1) (2008), 177-188.

21. S. Li and S. Stević, Riemann-Stieltjes operators between different weighted Bergman spaces, Bull. Belg. Math. Soc. Simon Stevin 15 (to appear).

22. S. Li and S. Stević, Weighted composition operators between $H^{\infty}$ and $\alpha$-Bloch spaces in the unit ball, Taiwanese J. Math. (accepted for publication).

23. L. Luo and S. I. Ueki, Weighted composition operators between weighted Bergman spaces and Hardy spaces on the unit ball of $\mathbb{C}^{n}, J$. Math. Anal. Appl. 326(1) (2007), 88-100.

24. B. D. MacCluer and R. Zhao, Essential norms of weighted composition operators between Bloch-type spaces, Rocky Mountain J. Math. 33(4) (2003), 1437-1458.

25. K. Madigan and A. Matheson, Compact composition operators on the Bloch space, Trans. Amer. Math. Soc. 347(7) (1995), 2679-2687.

26. S. Ohno, Weighted composition operators between $H^{\infty}$ and the Bloch space, Taiwanese J. Math. 5 (2001), 555-563.

27. S. Ohno, K. Stroethoff and R. Zhao, Weighted composition operators between Bloch type spaces, Rocky Mountain J. Math. 33 (2003), 191-215.

28. W. Rudin, Function theory in the unit ball of $\mathbb{C}^{n}$ (Springer-Verlag, New York, 1980).

29. J. H. Shi and L. Luo, Composition operators on the Bloch space, Acta Math. Sinica 16 (2000), 85-98. 
30. A. L. Shields and D. L. Williams, Bounded projections, duality, and multipliers in spaces of analytic functions, Trans. Amer. Math. Soc. 162 (1971), 287-302.

31. S. Stević, On an integral operator on the unit ball in $\mathbb{C}^{n}$, J. Inequal. Appl. 1 (2005), $81-88$.

32. S. Stević, Boundedness and compactness of an integral operator on a weighted space on the polydisc, Indian J. Pure Appl. Math. 37(6) (2006), 343-355.

33. S. Stević, Composition operators between $H^{\infty}$ and the $\alpha$-Bloch spaces on the polydisc, Z. Anal. Anwendungen 25(4) (2006), 457-466.

34. S. Stević, Boundedness and compactness of an integral operator on mixed norm spaces on the polydisc, Sibirsk. Mat. Zh. 48(3) (2007), 694-706.

35. S. Stević, On $\alpha$-Bloch spaces with Hadamard gaps, Abstr. Appl. Anal. 2007 (2007), 7 pp. (Article ID 39176).

36. S. Stević, Weighted composition operators between mixed norm spaces and $H_{\alpha}^{\infty}$ spaces in the unit ball, J. Inequal. Appl. 2007 (2007), 9 pp. (Article ID 28629).

37. S. Stević, On a new integral-type operator from the weighted Bergman space to the Bloch-type space on the unit ball, Discrete Dyn. Nat. Soc. 2008 (2008), 14 pp. (Article ID 154263).

38. S. Stević, Generalized composition operators from logarithmic Bloch spaces to mixednorm spaces, Util. Math. 77 (to appear)

39. S. Stević, Extended Cesàro operators between mixed-norm spaces and Bloch-type spaces in the unit ball, Houston J. Math. (to appear).

40. X. Tang, Extended Cesàro operators between Bloch-type spaces in the unit ball of $\mathbb{C}^{n}$, J. Math. Anal. Appl. 326(2) (2007), 1199-1211.

41. S. I. Ueki and L. Luo, Compact weighted composition operators and multiplication operators between Hardy spaces, Abstr. Appl. Anal. 2008 (2008), 11 pp. (Article ID 196498).

42. J. Xiao, Riemann-Stieltjes operators on weighted Bloch and Bergman spaces of the unit ball, J. Lond. Math. Soc. 70(2) (2004), 199-214.

43. S. Yamashita, Gap series and $\alpha$-Bloch functions, Yokohama Math. J. 28 (1980), 31-36.

44. S. Ye, Weighted composition operators between the little $\alpha$-Bloch space and the logarithmic Bloch, J. Comput. Anal. Appl. 10(2) (2008), 243-252.

45. K. Zhu, Spaces of holomorphic functions in the unit ball (Graduate Text in Mathematics 226, Springer, New York, 2005). 